\title{
Beginnings of the Cauchy problem for Einstein's field equations
}

\author{
Yvonne Choquet-Bruhat
}

\begin{abstract}
This is a brief account of results on the Cauchy problem for the Einstein equations starting with the early works of Darmois and Lichnerowicz and going up to the proofs of existence and uniqueness of solutions global in space and local in time, in Sobolev spaces, for the general equations either in vacuum or with classical sources.
\end{abstract}

\section{Introduction}

I was asked by the editors, whom I thank, to write a short article on the early works on the Cauchy problem for the book they were planning to celebrate the hundredth anniversary of Einstein's General Relativity. I accepted with pleasure, but I realized when I started to work on this project that it was more difficult than I thought. I have never been really interested in who did something first, and in fact it is often difficult to ascertain. Ideas have almost always one or several preliminaries, attribution of a name to the final flower is therefore somewhat arbitrary. The work of a true historian is long and difficult, I am not a historian. I often quote, not the first note touching a subject, but a later paper more complete and easier to find. The shortness of this article does not enable me to enter into details. Of course what I know best is my own work, it is part of my excuse for often quoting it. I apologize to all live or dead authors to whom I did not make enough deserved references. Other sources of information, including my own articles, can compensate my shortcomings.

2010 Mathematics Subject Classification. 35A01, 35L51, 35Q76, 83C05, 83C35.

Key words and phrases. Cauchy problem for Einstein equations, Einstein equations in vacuum and coupled to other fields, initial data, evolution, hyperbolic pde, ADM, gravitational waves, causality, Sobolev spaces. 


\section{Preliminary definitions}

The Einstein equations are a geometric system for a pair $(V, g)$, with $V$ an $n+1$ dimensional differentiable manifold, $n=3$ in the classical case, and $g$ a pseudo-Riemannan metric of Lorentzian signature. In vacuum they express the vanishing of the Ricci tensor

$$
\operatorname{Ricci}(g)=0,
$$

equivalently the vanishing of the Einstein tensor $(R(g)$ is the scalar curvature of $g$ )

$$
\operatorname{Einstein}(g):=\operatorname{Ricci}(g)-\frac{1}{2} g R(g)=0 .
$$

These equations are invariant under diffeomorphisms of $V$.

The Bianchi identities satisfied by the Riemann tensor imply, by two contractions, identities for the Einstein tensor which read ${ }^{1}$ in local coordinates $x^{\alpha}, \alpha=0,1, \ldots n$,

$$
\nabla_{\alpha} S^{\alpha \beta} \equiv \nabla_{\alpha}\left(R^{\alpha \beta}-\frac{1}{2} g^{\alpha \beta} R\right) \equiv 0,
$$

where $\nabla$ is the covariant derivative in the metric $g$

The vacuum Einstein equations constitute, from the analyst's point of view, a system of $\frac{(n+1)(n+2)}{2}$ second order quasilinear ${ }^{2}$ partial differential equations for the $\frac{(n+1)(n+2)}{2}, 10$ in the classical case $n=3$, coefficients $g_{\alpha \beta}$ of the metric $g$ in local coordinates. However these equations are not independent because of the above identities.

The Cauchy problem for a system of $N$ second order quasilinear partial differential equations with unknown $u$, a set of $N$ functions $u_{I}, I=1, \ldots, N$ on $R^{n+1}$,

$$
A_{J}^{I, \alpha \beta}(u, \partial u) \partial_{\alpha \beta}^{2} u_{I}=f_{J}(u, \partial u), \quad \partial_{\alpha}:=\frac{\partial}{\partial x^{\alpha}},
$$

is the search for a solution $u$ which takes, together with its set $\partial u$ of first order partial derivatives, given values $\bar{u}, \overline{\partial u}$ on a given $n$ dimensional submanifold $M$. The elements of the characteristic determinant of this system, for a function $u$ at a point $x$, are the second order polynomials in a vector $X$ :

$$
D_{J}^{I}(u, \partial u, X):=A_{J}^{I, \alpha \beta}(u, \partial u) X_{\alpha} X_{\beta},
$$

A submanifold with equation

$$
\phi\left(x^{\alpha}\right)=0
$$

is called characteristic at a point for the considered system and initial values $\bar{u}, \overline{\partial u}$ if the determinant with elements $\left(D_{J}^{I}\right)(\bar{u}, \overline{\partial u}, \partial \phi)$, polynomial of order $2 N$, vanishes at that point. The Cauchy-Kovalevski theorem says that if this system has analytic coefficients the Cauchy problem with analytic given

\footnotetext{
${ }^{1}$ We denote by $S$ the Einstein tensor. It is denoted by $G$ by some authors.

$2_{\text {i.e. linear with respect to second derivatives }}$
} 
initial data has one and only one analytic solution in a neighbourhood of $M$ if this submanifold is everywere non characteristic.

The Cauchy-Kowalevski theorem does not apply directly to the Einstein equations: their characteristic determinant is identically zero for any metric as can be foreseen from the identities satisfied by the Enstein tensor. The Cauchy problem for the Einstein equations is non standard and has led to interesting and difficult works.

\section{Analytic results}

Hilbert by Lagrangian methods and Einstein himself by approximation studies had been interested in what Einstein called "the force" of his equations, that is the generality of their solutions. However the history of exact results on the general Cauchy problem for the Einstein equations starts only in 1927 with the 47 pages book "Les équations de la gravitation Einsteinienne" by Georges Darmois [28], a professor of mathematics at the University of Paris ${ }^{3}$. Darmois considers (case $n=3$ ) a submanifold $M$ with equation $x^{0}=0$ and data on $M$ functions of the $x^{i}, i=1,2,3$, which will be the values on $M$ of $g_{\alpha \beta}$ and $\partial_{0} g_{\alpha \beta}$. The values on $M$ of the first and second partial derivatives $\partial_{\lambda \mu}^{2} g_{a \beta}$ are then determined by derivation of the data on $M$ except for the second transversal derivatives $\partial_{00}^{2} g_{a \beta}$. Darmois finds by straightforward computation the identities:

$$
\begin{aligned}
R_{i j} & \equiv-\frac{1}{2} g^{00} \partial_{00}^{2} g_{i j}+f_{i j}\left(g_{\alpha \beta}, \partial_{\lambda} g_{a \beta}, \partial_{\lambda h}^{2} g_{a \beta}\right) \\
R_{i 0} & \equiv \frac{1}{2} g^{j 0} \partial_{00}^{2} g_{i j}+f_{i 0}\left(g_{\alpha \beta}, \partial_{\lambda} g_{a \beta}, \partial_{\lambda h}^{2} g_{a \beta}\right) \\
R_{00} & \equiv-\frac{1}{2} g^{i j} \partial_{00}^{2} g_{i j}+f_{00}\left(g_{\alpha \beta}, \partial_{\lambda} g_{a \beta}, \partial_{\lambda h}^{2} g_{a \beta}\right.
\end{aligned}
$$

with $h$ Latin, i.e. space, indices ${ }^{4}$. The derivatives $\partial_{00}^{2} g_{\alpha 0}$ do not appear in any of these equations, as Darmois already foresaw because a change of coordinates preserving $M$ pointwise does not change $\partial_{00}^{2} g_{i j}$ on $M$, but permits to give arbitrary values to $\partial_{00}^{2} g_{\alpha 0}$.

If $g$ satisfies the vacuum Einstein equations, the equations $R_{i j}=0$ determine $\partial_{00}^{2} g_{i j}$ on $M$ if $g^{00}$ does not vanish there. Darmois concludes that significant discontinuities of the second derivatives of the gravitational potentials can occur across the submanifold $M, \phi\left(x^{\alpha}\right) \equiv x^{0}=0$, only if $g^{00}=0$ on $M$, that is if the hypersurface $M$ is tangent to the null cone

${ }^{3}$ In those times there was only one University of Paris. Sciences, letters, law and arts were housed in a building called the Sorbonne. G. Darmois was a man of varied interests. In 1948 he taught a course on probabilities which I attended. He was a member of the French Academy in the section "Astronomy".

${ }^{4}$ The text of Darmois is confusing because he gives Latin names to spacetime indices in (7) and Greek names in (8), (9), (10). Moreover in these three equations he assumes implicitly that the second time derivatives of the $g_{\alpha 0}$ are zero, in fact they do not appear in the equations. (See the very clear exposition in Lichnerowicz 1955 [49] p.29.) 
of the Lorentzian metric $g$, whose normals in the cotangent space satisfy the equation $g^{\alpha \beta} \partial_{\alpha} \phi \partial_{\beta} \phi=0$. This result, though not a proof of it, is in agreement with the propagation of gravitation with the speed of light, a fact already deduced by Einstein from approximations.

Darmois continues his study by remarking that, if $g^{00} \neq 0$, it is possible to extract $\partial_{00}^{2} g_{i j}$ from the equation $R_{i j}=0$ and, replacing these in the other equations by the so calculated expressions, obtain four equations which depend only on the initial data, equations which we now call the constraints; he mentions that they are the Gauss - Codazzi equations known from geometers and indicates that a solution of the equations $R_{i j}=0$ with data satisfying the constraints will satisfy the whole set, at least in the analytic case, due to the contracted Bianchi identities. Darmois recognizes that an analyticity hypothesis is physically unsatisfactory, because it hides the propagation properties of the gravitational field.

Darmois also studies, again in the analytic case, what initial data to give on a characteristic hypersurface $S_{0}$. He shows that they are the trace of the spacetime metric on the hypersurface and proves, in the analytic case, the existence of a local solution to the vacuum Einstein equation which is uniquely determined if one gives also its value on a 3-dimensional manifold $T$ transversal to $S_{0}$ or, what is equivalent for analytic functions, the values of all its derivatives at points of the intersection of $T$ and $S_{0}$. To show this, he uses adapted coordinates to decompose the problem into an evolution of some components of the metric to satisfy part of the Einstein equations, and the Bianchi identities to show that the remaining equation is also satisfied. The method used by Darmois does not extend to the non analytic case, though the introduction of the data of the trace of the metric on a second hypersurface, transversal to the characteristic one, but also characteristic in the non analytic case, has been successfully used, in particular in the nineties by Rendall. Before that, inspired by the general theorems of Leray for data with support "compact towards the past " 5 , the non analytic Cauchy problem was treated for data supported by a characteristic conoïd $[\mathbf{8}],[\mathbf{1 0}]{ }^{6}$

In the remainder of his book, after quoting the works of Droste and Schwarzschild on the solution with spherical symmetry, Darmois studies solutions with axial symmetry ${ }^{7}$.

Darmois had mentioned the geometric character of the constraints but he had worked in special coordinates, namely in Gaussian coordinates; that is, with timelines being geodesics normal to the initial manifold, the quantities that we call now ${ }^{8}$ lapse, $g_{00}$, and shift, $g_{0 i}$, are then equal respectively to one

\footnotetext{
${ }^{5}$ That is intersected along a compact set by the past of any point.

${ }^{6}$ For more recent works see papers by Cagnac and his students, in particular Dossa. Still more recent, Choquet-Bruhat, Chrusćiel and Martín-García, also Chrusćiel and collaborators.

${ }^{7}$ For other early work see J. Delsarte $[\mathbf{2 9}]$.

${ }^{8}$ Names given to the components of the tangent vector to the chosen timeline in the early sixties by J. A. Wheeler, a famous physicist coming from other branches of physics.
} 
and zero. Lichnerowicz, a bright student of the Ecole Normale Supérieure, had asked from Elie Cartan a subject for his thesis, and Cartan had proposed the proof of a conjecture he had on a property of symmetric spaces that he himself had not been able to prove for some time. Lichnerowicz proved it thirty years later, but when he met Darmois by chance in 1937, he was rather discouraged, and followed the suggestion of Darmois to work on problems of mathematical relativity, which were many and little considered at the time, instead of the too difficult problem proposed by Cartan. Lichnerowicz who was a man of varied interests, from algebra and differential geometry to theoretical physics and philosophy, followed Darmois' suggestion and completed quickly a thesis which appeared as a book [47]. In this book the Darmois computations on the Cauchy problem are extended to the case of a non constant lapse but the shift is kept zero. Lichnerowicz proposed the extension of the $3+1$ decomposition to a non zero shift to one of his two first students ${ }^{9}$, the author of this article, who did it through the use of the Cartan calculus in orthonormal frames, giving thus the general geometric formulas of the $n+1$ decomposition of the Ricci and Einstein tensor on a sliced manifold $M \times R$ in terms of the geometric elements: $t$-dependent induced metric $\bar{g}$ and extrinsic curvature $K$ of the slices $M \times\{t\}$. This led to a preliminary publication $[\mathbf{3 3}]$. However this formulation did not lead to an existence theorem for a non analytic solution of the evolutionary Cauchy problem and the full detailed article giving the geometric $n+1$ decomposition of the Einstein tensor in terms of $\bar{g}$ and $K$ was written only later [38]. Still later, motivated by the wish to find a quantification of the gravitational field, Arnowitt, Deser and Misner replaced in the $3+1$ decomposition the space-tensor $K$ by the space-tensor density $\pi$ conjugate of the space metric $\bar{g}$ in the Hamiltonian they were constructing for General Relativity [2] ${ }^{10}$. It is the decomposition with $\bar{g}$ and $K$ together with lapse and shift which is mainly used by mathematicians, though it is often called ADM formalism.

Lichnerowicz, as a student of Elie Cartan, had a formation of geometer. He was interested in geometric formulations and started the study of global problems [47], [49] ${ }^{11}$. He stated two and called them ${ }^{12}$ "propositions", $\mathrm{A}$ and B, for the Einsteinian spacetimes which he called regular. (The metric had to be $C^{2}$ by pieces with first derivatives satisfying "junction

Wheeler was an open and enthusiastic mind with no prejudice. He got conquered by General Relativity and its open possibilities. He believed in black holes and coined their name. He was also a gentleman, as a professor in Princeton he had many good students who admired and liked him.

${ }^{9}$ The other one was Yves Thiry, who worked on geometrical aspects and physical interpretation of the five dimensional unitary theory of Jordan, extension of Kaluza and Klein work.

${ }^{10}$ The Hamiltonian led Brill and Deser to the positive mass conjecture [5].

${ }^{11}$ The latter contains also a study of the 5 dimensional and the non symmetric unitary theories.

${ }^{12}$ Better named "conjectures". 
conditions".) ${ }^{13} \mathrm{~A}$ : introduction of matter sources in a vacuum spacetime can be done only in domains where this spacetime has singularities, B: the only complete vacuum spacetime with compact or asymptotically Euclidean space sections is flat. He proved $B$ in the case of stationary ${ }^{14}$ spacetimes; that is, the non existence of gravitational solitons. The Lichnerowicz result was much appreciated by Einstein who believed for physical reason that any complete asymptotically Euclidean vacuum Einsteinian spacetime should be Minkowski. The Christodoulou-Klainerman [26] global existence theorem (1989) has proven that the conjecture was false without stronger hypothesis than the ones originally made on the decay at infinity; that is, vanishing of the ADM mass as shown by Schoen and Yau [62], see also [70].

\section{Non analytic local existence, causality and gravitational waves}

It had already been stressed by Darmois on the one hand that analyticity was a bad physical hypothesis, on the other hand that a choice of coordinates was necessary to construct solutions of the Cauchy problem. The problem had interested Einstein himself and already [32] in 1918 he had used coordinates satisfying the flat spacetime wave equation to construct approximated solutions of the vacuum Einstein equations near the Minkowski spacetime.

Darmois $[\mathbf{2 8}]^{15}$ considers coordinates $x^{\lambda}$ which he calls "isothermes"; they satisfy the wave equations

$$
\square_{g} x^{\lambda} \equiv g^{\alpha \beta} \nabla_{\alpha} \partial_{\beta} x^{\lambda}=0 ;
$$

that is

$$
F^{\lambda} \equiv g^{\alpha \beta} \Gamma_{\alpha \beta}^{\lambda}=0
$$

Such coordinates are now called "harmonic" by analogy with solutions of the Laplace equations, or "wave" as suggested by Klainerman as being more appropriate.

By a straightforward concise and precise computation Darmois obtains the decomposition of the Ricci tensor of a pseudo Riemannian general metric as the sum of a second order system for the components of the metric and a term which vanishes identically in harmonic coordinates

$$
R_{\alpha \beta} \equiv R_{\alpha \beta}^{(h)}+L_{\alpha \beta}, \quad \text { with } L_{\alpha \beta} \equiv \frac{1}{2}\left\{g_{\alpha \lambda} \partial_{\beta} F^{\lambda}+g_{\beta \lambda} \partial_{\alpha} F^{\lambda}\right\}
$$

${ }^{13}$ The relevant condition is in fact that the Einstein equations are satisfied in a generalized sense: see [14].

${ }^{14}$ That is invariant under a timelike one parameter isometry group. The static case (timelines orthogonal to space sections) had been proven earlier by Racine [58], another student of Darmois.

${ }^{15}$ Darmois aknowledges prior consideration of harmonic coordinates by De Donder [31] and Lanczos [44]. 
where

$$
R_{\alpha \beta}^{(h)} \equiv-\frac{1}{2} g^{\lambda \mu} \partial_{\lambda \mu}^{2} g_{\alpha \beta}+P_{\alpha \beta}(g, \partial g) .
$$

with $P$ a quadratic form in the components of $\partial g$ whose coefficients are polynomials in the components of $g$ and its contravariant associate.

In harmonic coordinates the Einstein equations in vacuum reduce to the quasilinear quasi diagonal second order system $R_{\alpha \beta}^{(h)}=0$.

In the years shortly before the second world war great names in mathematics were working on the Cauchy problem for a second order equation of the type then called "hyperbolic normal", that is principal coefficients of Lorentzian signature. The Hadamard method of parametrix for solutions of linear equations seemed difficult to use for non linear equations. On the other hand a new method, energy estimates, introduced by Friedrichs and Lewy, was a subject of active research. An application of the energy estimates to the reduced vacuum Einstein equations enabled Stellmacher [65] to prove a uniqueness theorem for a local solution of the Cauchy problem for the reduced equations, with domain of dependence determined by the light cone; that is, a causality property. However, Stellmacher did not prove an existence theorem, in spite of a paper by Schauder $[\mathbf{6 0}]^{16}$, where was sketched an existence proof for a solution of one quasilinear second order equation by using energy estimates.

I was encouraged to look for the solution of the non analytic Cauchy problem for the Einstein equations in 1947 by Jean Leray who was giving a series of lectures on Cartan exterior differential systems, of which I was one of the few attendants. Leray gave me the name of Schauder as a reference but I found only his paper on hyperbolic system in two variables [61] written later, which I tried somewhat painfully to read, knowing no German. By chance, I fell on a paper by Sobolev [64], in French, which gives a construction of an elementary kernel for a second order linear hyperbolic equation in dimension $3+1$ without having to resort to a "finite part" parametrix nor to the method of descent for the case of even spacetime dimension, as did Hadamard. The Sobolev parametrix, whose definition extends to quasi diagonal second order systems, is constructed by solution of a system of integral equations on the characteristic conoïd. These equations, together with those defining the characteristic conoïd can be used to prove the existence of a solution of the Cauchy problem for the quasilinear reduced vacuum Einstein equations in a space of smooth functions. See [34] and references therein.

In the same paper I showed, using the Bianchi identities and a hyperbolic system satisfied by the harmonicity conditions, that the obtained solution satisfies the full Einstein equations if the initial data satisfy the constraints and that it is locally geometrically unique.

\footnotetext{
${ }^{16}$ Schauder, a collaborator and friend of J. Leray was a victim of the holocaust, having refused to follow Leray's advice to leave Germany, when it was still possible for Jews.
} 
These results ${ }^{17}$ were the subject of my thesis. Its jury included Lichnerowicz, Leray ${ }^{18}$ and Marcel Riesz ${ }^{19}$. I returned later to the construction of the elementary kernel of a tensorial linear system of second order hyperbolic differential equations [9] motivated by works on quantization in curved spacetime by A. Lichnerowicz who used a propagator, difference of the advanced and retarded elementary kernels $[\mathbf{5 0}]^{20}$. I pointed out that, being obtained by solving an integral equation on the light cone, the elementary kernel can be split into the sum of a measure supported by the light cone and a smooth function in its causal interior sum of a series of "diffusion terms", determined by integrations over characteristic cones with vertices at points of the previously considered cones $^{21}$. I studied the asymptotic behaviour of these terms.

Einstein, whom I met in 1951 at the Institute for Advanced Study in Princeton where I was a postdoc at the invitation of J. Leray, made me explain my thesis on the blackboard of his office; he congratulated me and invited me to knock at his door whenever I felt like it. I regret to have done it only a few times, in spite of his always kind welcome. Einstein was then working with his assistant Bruria Kaufmann on his last unified theory. His comments were very interesting, but the computations, which he himself enjoyed to do, were quite complicated and the theory rather deceptive ${ }^{22}$. I prefered to work at the extension to higher dimensions [36] of the formulas I had obtained in spacetime dimension 4 and follow the course of Leray on general hyperbolic systems. Though I also attended the Oppenheimer seminar on theoretical physics, where Einstein never came, I did not find there inspiration for personal work $^{23}$.

${ }^{17}$ See a report in Y. Bruhat [7].

${ }^{18}$ In fact, while I was still working on my thesis Leray was completing his momentum work on energy estimates and existence theorems for general hyperbolic systems, from which I could have deduced the result for the reduced Einstein equations, but Leray encouraged me warmly to pursue in the constructive direction I was following in the second order case.

${ }^{19}$ Present in Paris at that time. Darmois, an emeritus, could not belong to a thesis jury.

${ }^{20}$ See also Bryce DeWitt [30].

${ }^{21}$ For a lowering of the assumed regularity of the Lorentzian metric, see Klainerman and Rodnianski [43].

${ }^{22}$ Einstein tried at that time to interprete the antisymmetric part of the second rank tensor as electromagnetism. It appears that this last Einstein unified theory finds a renewal of interest with another intepretation (see Damour, Deser and McCarthy [27]).

${ }^{23} \mathrm{My}$ main memory of this seminar is a discussion of quantum vacua and the intervention of Wigner "but in vacuum there is nothing, nothing, there can be only one vacuum", Wigner, and also Einstein, lived in a time where the observed world could be thought to obey human scale logic. 


\section{Equations with sources}

The existence for classical sources in Special Relativity of a symmetric 2tensor $T$ representing energy, stresses and momentum densities which satisfy conservation laws was a motivation for Einstein in the choice ${ }^{24}$ of his non vacuum equations

$$
S_{\alpha \beta}=\kappa T_{\alpha \beta},
$$

with $\kappa$ a constant usually normalized to 1 by mathematicians. The problem is then the resolution of the coupled system of Einstein equations with sources and the conservation laws for these sources,

$$
\nabla_{\alpha} T^{\alpha \beta}=0
$$

with also eventually equations for fields other than gravitation, for instance Maxwell equations in presence of an electromagnetic field.

The Cauchy problem for the Einstein equations with sources splits again into constraints on initial data and an evolution problem for the reduced Einstein equations with sources. The treatment of the electrovacuum case is similar to vacuum and was solved simultaneously [35]. Solution in the cases of dust and perfect fluids without or with charge and zero conductivity were shown to admit a well posed Cauchy problem [39] using the Leray theory of hyperbolic systems [45]; relativistic fluids with infinite conductivity were analysed $[\mathbf{6}]$. They were seen to be what is now called Leray-Ohya $[\mathbf{4 6}]^{25}$ hyperbolic when Leray and Ohya proved well posedness of the Cauchy problem in Gevrey classes for some systems of differential equations with multiple characteristics. All these results obtained for barotropic fluids were extended by Lichnerowicz to fluids whose equation of state depends also on the entropy ${ }^{26}$ and assembled by him into a book [51] after a series of lectures he gave in Dallas at the invitation of Ivor Robinson. Relativistic fluids with finite conductivity were proved to be also Leray-Ohya hyperbolic [12]. Isotropic relativistic elasticity has been proven by Pichon to obey a Leray-Ohya hyperbolic system [57]. The hyperbolic character, Leray or Leray Ohya, holds for the Einstein equations coupled with any of the quoted sources. The equations of charged fluids with electromagnetic inductions are also Leray- Ohya hyperbolic, but their natural Maxwell tensor being non symmetric their coupling with Einstein equations is problematic ${ }^{27}$.

\footnotetext{
${ }^{24}$ With the help of his friend, the mathematician Grossmann.

${ }^{25}$ It was shown later by K.O. Friedrichs using general Lagrangian methods that fluids with infinite conductivity satisfy a first order symmetric hyperbolic system. See for instance $[\mathbf{1}]$.

${ }^{26}$ As suggested by A.Taub, on physical grounds.

${ }^{27}$ See [55]. Various symmetrizations have been proposed along the years, but their conservation laws lead to very unpleasant equations with unphysical interpretations. The physical answer -seems to be that at the scale where inductions play a role the gravitational field is negligible.
} 
Well posedness of the Cauchy problem was proved to be true for sources satisfying a Vlasov ${ }^{28}$, or a Boltzman ${ }^{29}$ equation with appropriate cross section.

\section{Constraints}

I said that it has long been known that geometric initial data for the vacuum Einstein equations on a spacelike submanifold $M$ are the two fundamental forms, induced metric $\bar{g}$ and extrinsic curvature $K$, and they must satisfy $n+1$ equations, the constraints. Surprisingly it took a long time to split these data into arbitrarily given quantities and unknowns which satisfy elliptic equations, as it was however reasonable to expect for unknowns on a space manifold and the Newtonian approximation of the Einstein equations. The first result in this direction was due to Racine [59]. He assumed, for $n+1=4$, the metric $\bar{g}$ to be conformally flat

$$
\bar{g}:=\phi^{4} e, \quad e \text { the Euclidean metric }
$$

and remarked that, if the trace $\bar{g}^{i j} K_{i j}$ of the extrinsic curvature $K$ vanishes and one sets

$$
P_{i j}=\phi^{2} K_{i j}
$$

the system of constraints for the equations with source of zero momentum splits into a first order linear system for $P$, independent of $\phi$, and a semi linear second order equation for $\phi$ with principal term the Laplacian $\Delta \phi$.

The study was taken anew by Lichnerowicz [48], replacing the Euclidean metric by a general Riemannian metric $\gamma$. He defines the traceless tensor $\tilde{K}_{i j}$ by

$$
\tilde{K}_{i j}=\varphi^{2}\left(K_{i j}-\frac{1}{3} \bar{g}_{i j} \tau\right), \quad \tau:=\bar{g}^{i j} K_{i j}
$$

The momentum constraint reads then as the linear system for $\tilde{K}$

$$
D_{i} \tilde{K}^{i j}=\frac{2}{3} \varphi^{6} \gamma^{i j} \partial_{i} \tau+\varphi^{10} J^{j}
$$

independent of $\varphi$ if the initial surface is maximal (he says "minimal") i.e. $\tau=0$, and if the momentum $J$ of the sources is zero. The Hamiltonian constraint reads then as a second order elliptic equation with only unknown $\varphi$ when $\tilde{K}$ and the matter density $\rho$ are known

$$
8 \Delta_{\gamma} \varphi-R(\gamma) \varphi+|\tilde{K}|_{\gamma}^{2} \varphi^{-7}+\left(\rho-\frac{2}{3} \tau^{2}\right) \varphi^{5}=0
$$

\footnotetext{
${ }^{28}$ Case of particles with a given rest mass and no charge:

Y. Choquet-Bruhat [17].

Case of particles with electric charge and arbitrary (positive) rest masses using a weight factor to obtain convergent integrals:

Y. Choquet-Bruhat [18].

${ }^{29}$ D. Bancel $[\mathbf{3}]$

D. Bancel and Y. Choquet-Bruhat [4]
} 
Lichnerowicz constructs a class of exact initially static data for the $N$ body problem with supports in domains $D_{I}, I=1, \ldots n$, and matter densities $\mu_{I}$ by taking $\gamma=e$, assuming $J=0, \tau=0$ and taking $\tilde{K}=0$ as a solution of the momentum constraint. The system of constraints reduces then to one elliptic non linear equation with principal term the Euclidean Laplace operator

$$
\Delta \varphi=f(\varphi) \text { with } f(\varphi)=0 \text { in vacuum and } f(\varphi) \equiv-\frac{1}{8} \mu_{I} \varphi^{5} \text { in } D_{I} .
$$

Using the potential formula Lichnerowicz solves this equation by iteration, showing the convergence of the series for small enough $\mu_{I}$. The problem of meaningful non static solutions of the momentum constraint remained unsolved.

It is only in 1961, writing an article on the Cauchy problem, for the book edited by Louis Witten [7] and inspired by a paper of D. Sharp [63] on possible constraints for the arbitrary quantities of the "thin sandwich conjecture" of J. A. Wheeler, namely lapse and shift, which did not lead to an elliptic system, that I realized that such an elliptic system can be written for the corresponding spacetime densities $\mathcal{G}^{00}$ and $\mathcal{G}^{i 0}$ by using the splitting of the Einstein equations obtained through the harmonic gauge. A. Vaillant-Simon [68] constructed a solution of this system close to the Minkowski spacetime.

In 1971, I wrote an elliptic, but not quasidiagonal [23], system for geometric data on an arbitrary spacelike manifold which stimulated the interest of J. York, then a student of J. A. Wheeler, in the constraint problem. York remarked that the assumption "maximal" on the initial manifold made for conformally formulated constraints can be replaced by constant mean extrinsic curvature $[\mathbf{7 1}]^{30}$ and he introduced weights for the sources $\rho$ and $J$, physically justified at least for electromagnetic sources and dimension $n=3$. He thus obtained the linear momentum constraint independent of $\varphi$

$$
D_{i} \tilde{K}^{i j}=\tilde{J}^{i} .
$$

The Hamiltonian constraint becomes then the nonlinear elliptic equation with only unknown $\varphi$ when $\gamma$ is chosen, $\tilde{K}$ computed and $\tilde{\rho}$ is known

$$
8 \Delta_{\gamma} \varphi-R(\gamma) \varphi+|\tilde{K}|_{\gamma}^{2} \varphi^{-7}+\tilde{\rho} \varphi^{-3}-\frac{2}{3} \tau^{2} \varphi^{5}=0 .
$$

A decomposition theorem for symmetric 2-tensors, linked to the fact that Lie derivatives of vector fields span the $L^{2}$ dual of divergence free symmetric 2 -tensors, had been known for some time. It leads to the writing $[\mathbf{7 2}]^{31}$ of the general solution of the momentum constraint, when $\tau$ is constant, under the form, with $U$ an arbitrary given traceless symmetric 2 tensor

${ }^{30}$ The constant non zero situation was neglected by previous authors who were only interested in the asymptotically Euclidean manifolds where it does not occur.

${ }^{31}$ See J W. York $[\mathbf{7 2}]$ and references therein. 


$$
\begin{aligned}
& \tilde{K}^{i j}=\left(\mathcal{L}_{\gamma, \text { conf }} X\right)^{i j}+U^{i j}+\frac{1}{3} \gamma^{i j} \tau, \\
& \left(\mathcal{L}_{\gamma, \text { conf }} X\right)^{i j}:=D^{i} X^{j}+D^{j} X^{i}-\frac{2}{3} \gamma^{i j} D_{k} X^{k} .
\end{aligned}
$$

The vector field $X$ is then solving the linear system

$$
\Delta_{\gamma, \text { conf }} X:=D \cdot\left(\mathcal{L}_{\gamma, \text { conf }} X\right)=-D \cdot U+\tilde{J} .
$$

which can be shown to be equivalent to a linear elliptic second order operator for $X$, to which known theorems can be applied.

The conformal method gave to the Hamiltonian constraint on a manifold a geometric comparatively simple form but non linear with no known generic solution. I thought of applying to it the Leray-Schauder degree theory. I brought to Leray in 1962 a note about its solution in Hölder spaces for publication in the C.R. of the French Academy of Sciences. Leray remarked that my result would hold for more general equations, and suggested we publish jointly the general result. It was for me a great honor. Leray wrote [25] very fast for compact manifolds this note which introduces sub and super solutions, and refused to cosign the note solving the particular case of the Hamiltonian constraint which I wrote shortly afterwards [24]. I obtained later the result for asymptotically Euclidean manifolds in weighted Hölder spaces [20]. A large amount of work has been devoted since that time to the solution of the constraints expressed as the elliptic semilinear system obtained by the conformal method on a constant mean curvature initial manifold, and using weighted Sobolev spaces. Progress has been made in lifting the constant mean curvature hypothesis and weakening the regularity, but there is space for further work.

\section{Local existence and global uniqueness}

In the beginning of the seventies the geometric character of the Cauchy problem for the Einstein equations was well understood ${ }^{32}$. The global in space, local in time existence was known for the classical sources mentioned above, at least for compact or asymptotically Euclidean manifolds, apart from lessening the regularity required of data and abandoning the constant mean curvature hypothesis of the initial manifold. Local uniqueness, up to diffeomorphisms, of a solution of the evolution of geometric data satisfying the constraints was also known, but the question of a global isomorphism between solutions was open. In fact, though known to specialists, the geometric local existence and uniqueness did not have in the literature a concise and precise formulation. I convinced Geroch we write up proper definitions for the geometric local theorem [22] before publishing our global geometric uniqueness proof.

\footnotetext{
${ }^{32}$ Let us quote in addition to previously quoted papers the following ones which point out some problems still open: J. A. Wheeler [69], R. Penrose [53], S. Hawking [41].
} 
A fundamental notion for the study of global solutions of linear hyperbolic systems of arbitrary order had been introduced in 1952 by J. Leray. He had defined what he called "time like paths" for such systems and defined global hyperbolicity as compactness (in the space of paths) of any, non vacuum, set of timelike paths joining two arbitrary points. This general definition applies in particular to Lorentzian manifolds. In this case global hyperbolicity was proved (Choquet-Bruhat 1967) to be equivalent to the strong causality defined by Penrose 1967 added to the compactness of the spacetime domains defined by intersections of past and future of any two spacetime points (see definitions and proof in the Hawking and Ellis book [42] of 1973).

In 1969, the local existence was completed [22] by a geometric global uniqueness result, namely existence and uniqueness, up to isometries, in the class of globally hyperbolic spacetimes, of a maximal ${ }^{33}$ Einsteinian development of given initial data. Geroch and myself had met and discussed at the "Batelle rencontres 1967" organized by J. A. Wheeler and C. DeWitt. We obtained a complete proof during a visit we both made in London ${ }^{34}$. In 1970 Geroch proved the following very useful criterium: global hyperbolicity is equivalent to the existence of a "Cauchy surface", that is a 3-manifold cut once and only once by any timelike curve without end point.

The geometric global uniqueness result, first proved in the vacuum case, extends to Einstein equations with sources which have a well posed causal Cauchy problem; that is, in particular, satisfy Leray or Leray-Ohya hyperbolic systems.

\section{Global existence and singularities}

Generic problems of global existence or formation of singularities were, towards the end of the sixties, mainly open. They became for analysts and geometers interested in the modeling of the world we live in, a vast field of research. It led to new definitions, conjectures, remarkable results, and new open problems.

As a remark for the references, see footnote ${ }^{35}$.

\section{References}

[1] A. M. Anile "Relativistic fluids and magnetofluids" Cambridge University Press, 1989.

[2] R. Arnowitt, S. Deser and C. Misner "The Dynamics of General Relativity" in "Gravitation, an Introduction to current research", L. Witten ed. Plenum 1962.

[3] D. Bancel "Problème de Cauchy pour l'équation de Boltzmann en Relativité Générale" Ann. Inst. Poincaré XVIII n ${ }^{0} 3$ 263-284 1973

$33_{\text {i.e. }}$ which cannot be isometrically embedded into a larger Einsteinian spacetime.

${ }^{34}$ I had obtained in 1968 a global uniqueness theorem, but restricted to complete Einsteinian spacetimes.

${ }^{35}$ Y Bruhat, Y. Choquet-Bruhat and Y. Fourès-Bruhat are the same person, daughter of the physicist Georges Bruhat. 
[4] D. Bancel and Y. Choquet-Bruhat "Existence, uniqueness and local stability for the Einstein-Maxwell-Boltzman system" Com. Math. Phys. Vol. 33, Issue 2. 83-96. 1973.

[5] D. R. Brill and S. Deser Ann. Phys. (N. Y.) 508401968

[6] Y. Bruhat "Fluides relativistes de conductivité infinie" Astronautica Acta 6 354-355 1961

[7] Y. Bruhat " The Cauchy problem" in "Gravitation, an introduction to current research" Louis Witten ed. Wiley 1962

[8] Y. Bruhat "Problème des conditions initiales sur un conoïde caractéristique C.R. Acad. Sci 256, 371-373, 1963.

[9] Y. Bruhat "Sur la théorie des propagateurs" Annali di Matematica Serie IV, tomo LXIV -1964.

[10] F. Cagnac "Problème de Cauchy sur les hypersurfaces caractéristiques des équations d'Einstein du vide" C.R. Acad Sci 2621966

[11] F. Cagnac "Problème de Cauchy sur un conoïde caractéristique pour les équations d'Einstein du vide" Annali di matemetica pura e applicata 1975.

[12] Y. Choquet-Bruhat "Etude des équations des fluides chargés relativistes inductifs et conducteurs" Com. Math. Phys 3 334-357 1966.

[13] Y. Choquet-Bruhat "Hyperbolic partial differential equations on a manifold" in C. DeWitt and J.A. Wheeler ed "Batelle rencontres 1967 in Mathematics and Physics"

[14] Y. Choquet-Bruhat" Espaces Einsteiniens généraux, chocs gravitationnels" Ann. Inst. Poincaré, $8 \mathrm{n}^{\circ} 4$, 327-338, 1968.

[15] Y. Choquet-Bruhat "Un théorème global d'unicité pour les solutions des équations d'Einstein" Bul. Soc. Math. France 96 181-192, 1968.

[16] Y. Choquet-Bruhat "New elliptic system and global solutions for the constraints equations in General Relativity" Com. Math. Phys. 21 211-218, 1971.

[17] Y. Choquet-Bruhat "Solution du problème de Cauchy pour le système intégrodifferentiel d'Einstein Liouville" Ann..Inst. Fourier XXI 3 181-203 1971.

[18] Y. Choquet-Bruhat "Existence and uniqueness for the Einstein-Maxwell-Liouville system" Volume in honor of Professor Petrov's 60th birthday Kiev 1971.

[19] Y. Choquet-Bruhat "Solution globale du problème des contraintes sur une variété compacte" C.R. Acad Sci. 274 682-684 1972.

[20] Y. Choquet-Bruhat "Solution of the problem of constraints on open and closed manifolds" J. Gen. Rel and Grav. 5 45-64 1974.

[21] Y. Choquet-Bruhat and R. Geroch "Probleme de Cauchy intrinsèque en Relativité générale" C. R. Acad. Sci. 269 746-748, 1969.

[22] Y. Choquet-Bruhat and R. Geroch "Global aspects of the Cauchy problem in General Relativity" Comm. Math. Phys. 14, 329-335, 1969.

[23] Y. Choquet-Bruhat Com. Math. Phys. 21 211-218, 1971.

[24] Y. Choquet-Bruhat " Global solutions of the equations of constraints in general relativity on closed manifolds", Symposia Mathematica, Vol. XII, 317-325. Academic Press, London, 1973.

[25] Y. Choquet-Bruhat et J. Leray "Sur le problème de Dirichlet quasi-linéaire d'ordre 2" C R. Acad. Sci 274 81-85 1972

[26] D. Christodoulou and S. Klainerman. "The Global Nonlinear Stability of the Minkowski Space" Princeton University Press. 1993.

[27] T. Damour, S. Deser and J. McCarthy. Phys. Rev. D (3) 47, 1541-1556, 1993.

[28] G. Darmois "Les équations de la gravitation Einsteinienne" Memorial des Sciences mathématiques Gauthier Villars 1927.

[29] J. Delsarte "Sur les ds² d'Einstein à symétrie axiale' Hermann 1934.

[30] B. DeWitt."Quantization of geometry" in Les Houches 1963, Gordon and Breach

[31] T. De Donder "La gravifique Einsteinienne" Gauthier Villars Paris 1921.

[32] A. Einstein. Sitzgsb. 1918.

[33] Y. Fourès-Bruhat C. R. Acad Sci. Paris 1948 
[34] Y. Fourès-Bruhat "Théorèmes d'existence pour certains systèmes d'équations aux dérivées partielles non linéaires" Acta Mathematica 88, 42-225, 1952.

[35] Y. Fourès-Bruhat "Théorème d'existence et d'unicité dans les théories relativistes de l'électromagnétisme" C.R. Acad.Sci. 2321951.

[36] Y. Fourès-Bruhat "Résolution du problème de Cauchy pour des équations hyperboliques du second ordre non linéaires" Bull. Soc. Math. France 81, 225-288 1953

[37] Y. Fourès-Bruhat "Solution élémentaire d'équations ultrahyperboliques" J. Math. Pures et Ap 121 277-289 1955.

[38] Y. Fourès-Bruhat "sur l'intégration des équations de la Relativité Générale" J. Rat. Mech. and Anal. 5 951-966 1956.

[39] Y. Fourès-Bruhat" Theorèmes d'existence en mécanique des fluides relativistes" Bull. Soc. France 86, 155-175, 1958.

[40] R. Geroch "The domain of dependence" J. Math. Phys 11 437-439 1970

[41] S. Hawking. Proc. Roy. Soc. 294 A 511, 1966.

[42] S. W. Hawking and G.R.F Ellis "The large scale structure of spacetime" Cambridge university press 1973.

[43] S. Klainerman and I. Rodnianski. "A Kirchoff-Sobolev parametrix for the wave equation and applications" J. Hyperbolic Differ. Equ. 4, no. 3, 401-433, 2007.

[44] K. Lanczos "Ein vereinfachendes Koordinatensystem für die Einsteinschen Gravitationsgleichungen" Phys. Zeitschrift 23 537-539, 1922.

[45] J. Leray "Hyperbolic differential systems" Mimeographed Notes, IAS Princeton 1953.

[46] J. Leray and Y. Ohya Math Annalen 162, 228-236 1968.

[47] A. Lichnerowicz "Problèmes globaux en mécanique relativiste", Hermann 1939.

[48] A. Lichnerowicz. "L'intégration des équations de la gravitation relativiste et le problème des n corps" J. Math. pures et App. 37-63, 1944.

[49] A. Lichnerowicz "Théories relativistes de la gravitation et de l'électromagnétisme" Masson, Paris 1955.

[50] A. Lichnerowicz "Propagateurs et commutateurs en Relatitité Générale", publications mathématiques de l'IHES, 1, 1961

[51] A. Lichnerowicz "Relativistic fluids and magneto fluids" Benjamin 1967.

[52] N. O'Murchada and J. W. York "The initial value problem of General Relativity" Phys. Rev. D 10 428-446 1974.

[53] R. Penrose. Phys. Rev. Let. 14 57, 1965.

[54] R. Penrose "Structure of spacetime" in C. DeWitt and J.A. Wheeler ed "Batelle rencontres 1967 in Mathematics and Physics"

[55] M. Q. Pham "Etude électrodynamique et thermodynamique d'un fluide relativiste chargé" J. Rat. Mech. Anal. 5, 473-538,1956.

[56] G. Pichon. "Etude relativiste de fluides visqueux et chargés" Ann. Inst. Poincaré II $\mathrm{n}^{0} 11965$.

[57] G. Pichon "Théorèmes d'existence pour les équations des milieux élastiques" J. Math. Pures. et App 45 3395-409 1966

[58] Ch. Racine C.R. Acad Sci. Paris 1931.

[59] Ch. Racine "Le problème des $n$ corps dans la théorie de la Relativité " Thèse Paris 1934, Gauthier Villars.

[60] J. Schauder Fundamenta mathematicae, 24 213-246 1935.

[61] J. Schauder Comm. Math. Helv.9 1936-1937

[62] R. Schoen and S.-T. Yau. "On the proof of the positive mass conjecture in general relativity" Comm.Math.Phys. 65. 45-76, 1979.

[63] D. Sharp "One and two surfaces formulation of the boundary value problem for the Einstein- Maxwell equations" thesis Princeton University 1961

[64] S. Sobolev "Methode nouvelle à résoudre le problème de Cauchy pour les équations linéaires hyperboliques normales" Rec. Math. Moscou N. s. 1936.

[65] K. Stellmacher. Math. Annalen 115, 1938. 
[66] A.H. Taub Phys. Rev 74 328-334 1948

[67] A.H. Taub J. Rat. Mech. Anal. 3 312-324 1959.

[68] A. Vaillant-Simon, J. maths pures et App 48; 1-90, 1969.

[69] J. A. Wheeler in "Relativity, Groups and Topoloy", B. and C. DeWitt ed. Gordon and Breach 1964

[70] E. Witten. "A new proof of the positive energy theorem" Comm.Math.Phys. 80. 381402, 1981.

[71] J. W. York "Role of conformal 3 geometry in the dynamics of gravitation" Phys. Rev. lett. 28 1082.1972.

[72] J W. York "Decomposition of symmetric 2-tensors in the theory of gravitation" Annales de l'IHP A 4, 319-331 1974.

Institut des Hautes Études Scientifiques, Bures-sur-Yvette, France E-mail address: ycb@ihes.fr 